Enfermagem Brasil 2018;17(6):606-18

https://doi.org/10.33233/eb.v17i6.2063

\title{
ARTIGO ORIGINAL \\ Feridas neoplásicas em pacientes com câncer de mama
}

Débora Thaise Freires de Brito*, Irys Karla Cosmo Pereira*, Glenda Agra** ${ }^{* *}$, Elton de Lima

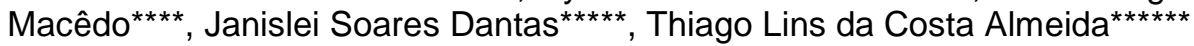

*Enfermeira, Universidade Federal de Campina Grande/UFCG, campus Cuité, Residente pelo Programa de Residência Multiprofissional em Saúde da Família e Comunidade, João Pessoa/PB, **Enfermeira, Universidade Federal de Campina Grande/UFCG, campus Cuité, Bananeiras/PB, ***Enfermeira, Doutoranda pelo Programa de Pós-Graduação em Enfermagem da Universidade Federal da Paraíba/UFPB, Docente do Curso de Graduação em Enfermagem da Universidade Federal de Campina Grande/UFCG, campus Cuité. João Pessoa/PB, **** Enfermeiro, Universidade Federal de Campina Grande/UFCG, campus Cuité, Recife/PB,

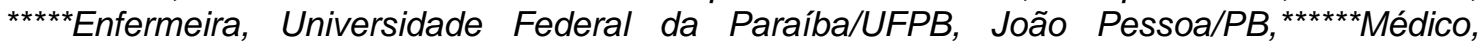
Universidade Federal da Paraíba/UFPB, João Pessoa/PB

Recebido em 21 de janeiro de 2018; aceito em 20 de junho de 2018.

Endereço para correspondência: Débora Thaíse Freires de Brito, Avenida Aluísio França, 157, Manaíra, 58038-060 João Pessoa PB, E-mail: deborathaise_@hotmail.com; Irys Karla Cosmo Pereira: iryscosmopereira@hotmail.com; Glenda Agra: glendaagra@outlook.com; Elton de Lima Macêdo: eltoneltonlm@hotmail.com; Janislei Soares Dantas: janisleisd@gmail.com; Thiago Lins da Costa Almeida: linsalmeida@gmail.com

\section{Resumo}

Objetivo: Traçar o perfil sociodemográfico, clínico e terapêutico de pacientes com feridas neoplásicas, decorrentes do câncer de mama. Métodos: Trata-se de um estudo documental, retrospectivo e descritivo, com abordagem quantitativa, em que foram utilizados dados secundários-sociodemográficos, clínicos e terapêuticos - extraídos de prontuários de pacientes com feridas neoplásicas, no intervalo de janeiro de 2009 a dezembro de 2015. Resultados: Fizeram parte deste estudo 150 prontuários, destes, 147 eram mulheres $(98,0 \%)$, com idade entre 20 e 59 anos $(66,0 \%)$, casadas $(41,3 \%)$, ensino fundamental incompleto $(24,0 \%)$, agricultoras $(25,3 \%)$ e aposentadas $(24,7 \%)$. O tipo histológico carcinoma ductal invasivo se sobressaiu em 131 pacientes $(87,3 \%)$, cujas lesões apresentavam aspecto de ulceração infiltrante $(30,7 \%)$, dor $(15,3 \%)$, sangramento $(3,3 \%)$, odor $(2,7 \%)$, exsudato $(9,3 \%)$ e sinais de infecção $(2,7 \%)$. Quanto à modalidade terapêutica, 63 pacientes $(42,0 \%)$ se submeteram à mastectomia total, $102(68,0 \%)$ realizaram radioterapia e $125(83,3 \%)$ quimioterapia. Conclusão: Evidenciou-se a necessidade de implementar políticas públicas no tocante à prevenção do câncer de mama, a fim de diminuir o risco para adoecimento; educação em saúde para esses pacientes, visando reduzir complicações locais e sistêmicas; e criar estratégias efetivas para o registro das informações sociodemográficas, clínicas e terapêuticas completas do paciente em prontuários eletrônicos ou convencionais pelos profissionais de saúde.

Palavras-chave: epidemiologia, enfermagem oncológica, neoplasias cutâneas.

\section{Abstract \\ Neoplastic wounds in breast cancer patients}

Objective: To describe the sociodemographic, clinical and therapeutic profile of patients with neoplastic wounds, due to breast cancer. Methods: It is a retrospective and descriptive documentary study with a quantitative approach, in which secondary data were used - sociodemographic, clinical and therapeutic - extracted from medical records of patients with neoplastic wounds, between January 2009 and December 2015. Results: The sample consisted of 150 medical records, of which 147 were women (98.0\%), aged between 20 and 59 years $(66.0 \%)$, married $(41.3 \%)$ and had incomplete elementary school $(24.0 \%)$, women farmers $(25.3 \%)$ and retirees $(24.7 \%)$. The histological type invasive ductal carcinoma was prominent in 131 patients $(87.3 \%)$, with lesions presenting with infiltrating ulceration $(30.7 \%)$, pain $(15.3 \%)$, bleeding $(3.3 \%)$, odor $(2.7 \%)$, exudate $(9.3 \%)$ and signs of infection $(2.7 \%)$. Regarding the 
therapeutic modality, 63 patients $(42.0 \%)$ underwent total mastectomy, $102(68.0 \%)$ underwent radiotherapy and $125(83.3 \%)$ underwent chemotherapy. Conclusion: The need to implement public policies regarding the prevention of breast cancer was evidenced in order to reduce the risk of becoming ill; health education for these patients, aiming to reduce local and systemic complications; and to create effective strategies for the registration of the patient's complete sociodemographic, clinical and therapeutic information in electronic or conventional medical records by health professionals.

Key-words: epidemiology, nursing oncology, skin neoplasms.

\section{Resumen \\ Heridas neoplásicas en pacientes con cáncer de mama}

Objetivo: Trazar el perfil sociodemográfico, clínico y terapéutico de pacientes con heridas neoplásicas, resultantes del cáncer de mama. Métodos: Se trata de un estudio documental, retrospectivo y descriptivo, con abordaje cuantitativo, en el que se utilizaron datos secundarios - sociodemográficos, clínicos y terapéuticos - extraídos de prontuarios de pacientes con heridas neoplásicas, en el intervalo de enero de 2009 a diciembre de 2015. Resultados: La muestra fue compuesta por 150 prontuarios, de éstos, 147 eran mujeres (98,0\%), con edad entre 20 a 59 años $(66,0 \%)$, casadas $(41,3 \%)$, con enseñanza fundamental incompleta (24), $0 \%)$, agricultoras $(25,3 \%)$ y jubiladas $(24,7 \%)$. El tipo histológico carcinoma ductal invasivo se sobresalió en 131 pacientes $(87,3 \%)$, en que las lesiones presentaban aspecto de ulceración infiltrante $(30,7 \%)$, dolor $(15,3 \%)$, sangrado $(3,3 \%),(2,7 \%)$, exudado $(9,3 \%)$ y signos de infección $(2,7 \%)$. En cuanto a la modalidad terapéutica, 63 pacientes $(42,0 \%)$ se sometieron a la mastectomía total, $102(68,0 \%)$ realizaron radioterapia y $125(83,3 \%)$ quimioterapia. Conclusión: Se evidenció la necesidad de implementar políticas públicas en cuanto a la prevención del cáncer de mama, a fin de disminuir el riesgo para enfermarse; educación en salud para estos pacientes, con el fin de reducir las complicaciones locales y sistémicas; y crear estrategias efectivas para el registro de las informaciones sociodemográficas, clínicas y terapéuticas completas del paciente en prontuarios electrónicos o convencionales por los profesionales de salud.

Palabras-clave: epidemiología, enfermería oncológica, neoplasias cutáneas.

Introdução

O câncer de mama é o tipo de neoplasia mais incidente e que causa maior mortalidade na população feminina em todo o mundo, tanto em países em desenvolvimento quanto em países desenvolvidos, com uma incidência de 1,7 milhões de novos casos ou $11,9 \%$ de todos os cânceres [1].

No Brasil, segundo o Instituto Nacional do Câncer (INCA), a estimativa para o biênio 2016/2017 apontou que o câncer de mama foi o mais incidente na população feminina, com cerca de 58 mil casos novos com um risco estimado de 56,20 casos a cada 100 mil mulheres, reforçando a magnitude do problema no país. Para estes mesmos anos, na região Nordeste, estimaram-se 11.190 casos novos de câncer de mama com um risco de 38,74 casos a cada 100 mil mulheres e na Paraíba, estimaram-se 800 casos novos de câncer de mama com risco de 39,50 casos a cada 100 mil mulheres [1].

O câncer de mama é considerado uma doença heterogênea com relação à clínica, derivado de variações genéticas e morfológicas. A maioria desses tumores se origina no epitélio ductal (cerca de $90 \%$ ) e é conhecido como carcinoma ductal invasivo, contudo existem mais de 20 subtipos diferentes da doença. Dessa forma, outros subtipos podem ser diagnosticados como o lobular, tubular, mucinoso, medular, micropapilar, papilar, entre outros [1-2].

Vale salientar, que uma das complicações do câncer de mama é o aparecimento de feridas neoplásicas resultante da infiltração das células malignas do tumor nas estruturas da pele, levando consequentemente à quebra da sua integridade, com posterior formação de uma ferida evolutivamente exofítica, decorrente da proliferação celular descontrolada que 0 processo de oncogênese provoca [3].

Embora não existam dados que confirmem a incidência fidedigna de feridas neoplásicas decorrentes do avanço do câncer, pode-se realizar uma estimativa sobre essa associação. Diante disso, um estudo verificou que $5 \%$ a $10 \%$ dos pacientes com câncer desenvolvem feridas neoplásicas, seja em decorrência do tumor primário ou de tumores metastáticos, sobretudo nos últimos seis a 12 meses de vida [4]. 
Essas feridas quando acometem a mama constituem mais um agravo na vida do paciente oncológico, pois, progressivamente, tornam-se friáveis, dolorosas, exsudativas, fétidas, podem desfigurar o corpo e provocar no paciente distúrbio da autoimagem e desgaste psicológico, bem como sensação de desamparo, humilhação, isolamento social e tristeza [5-6].

Diante dessas circunstâncias, vale ressaltar que a equipe multiprofissional deve estar empenhada em cuidar do paciente com câncer de mama que desenvolve ferida neoplásica. Nessa conjuntura, o enfermeiro é um dos membros responsável em avaliar o paciente e a lesão, bem como realizar os curativos adequados e acompanhar o paciente até o desfecho (alta ou óbito). Diante disso, é primordial que o enfermeiro tenha conhecimento sobre os produtos, substâncias, coberturas e medicamentos específicos mais indicados para controle dos sinais e sintomas, a realidade econômica do paciente e de seus familiares e da instituição em que está hospitalizado para assim melhor intervir [7].

Nessa perspectiva, a motivação pela temática surgiu a partir da escassez de estudos que contribuíssem para melhorar a qualidade da assistência prestada, uma vez que pesquisas científicas que abordam dados epidemiológicos, descritivos, clínicos e terapêuticos de pacientes com feridas neoplásicas são incipientes no Brasil.

$\mathrm{Na}$ intenção de aprofundar o conhecimento acerca da temática, emergiu a seguinte questão: Qual o perfil sociodemográfico, clínico e terapêutico de pacientes com feridas neoplásicas decorrentes do câncer de mama que foram atendidos em um hospital de referência na Paraíba? Esse questionamento representou o ponto de partida para 0 desenvolvimento da pesquisa por considerar que há necessidade de conhecer a clientela para a qual se presta diversas formas de cuidados e, assim, direcionar o seu atendimento.

Diante disso, o objetivo do estudo foi traçar o perfil sociodemográfico, clínico e terapêutico de pacientes com feridas neoplásicas decorrentes do câncer de mama.

\section{Material e métodos}

Trata-se de um estudo documental, retrospectivo e descritivo, com abordagem quantitativa, no qual foram utilizados dados secundários extraídos de prontuários de pacientes com câncer do Serviço de Arquivo Médico e Estatística de um hospital filantrópico do município de Campina Grande, na Paraíba.

A coleta de dados foi realizada durante o período de agosto de 2015 a abril de 2016, em que foram analisados 8.360 prontuários de pacientes com câncer. Após a leitura destes, foram identificados 150 prontuários que atendiam aos critérios de inclusão, constituindo-se, assim, a amostra final do estudo, sendo esta do tipo intencional.

Os critérios de inclusão adotados para a amostra foram: prontuários de pacientes com idade igual ou maior que 18 anos, que possuíssem o diagnóstico de câncer de mama, independente da fase da doença, com presença de ferida neoplásica exofítica na mama, no espaço temporal de 2009 a 2015. Este intervalo é justificado devido à primeira publicação do Manual para Cuidados de Pacientes com Feridas Tumorais do Instituto Nacional do Câncer [3] ter datado de 2007 e por isso, levou-se em consideração pelo menos um intervalo mínimo de dois anos para que o manual apresentasse um alcance à rede de serviço público do país. Como critério de exclusão, adotaram-se prontuários que apresentaram preenchimento dos dados com letras ilegíveis ou em branco.

Para coleta de dados, utilizou-se um roteiro estruturado adaptado do Manual do Instituto Nacional do Câncer, [3] que aborda recomendações acerca do controle de sinais e sintomas de feridas tumorais. Ao finalizar a coleta, os dados foram transferidos para o Microsoft Word, dispostos em tabelas, e em seguida foi realizada a análise descritiva das variáveis do estudo.

A pesquisa foi aprovada pelo Comitê de Ética em Pesquisa do Hospital Universitário Alcides Carneiro sob CAAE no 42918715.1.0000.5182 e obedeceu às recomendações éticas dispostas nas diretrizes e normas regulamentadoras para pesquisas envolvendo seres humanos, estabelecidas na Resolução no466/2012 do Conselho Nacional de Saúde em vigor no país. Também foram respeitadas as observações éticas presentes no Código de Ética dos Profissionais de Enfermagem - Resolução № 311/2007 do Conselho Federal de Enfermagem (COFEN), capítulo III, que trata das responsabilidades, deveres e proibições referentes ao ensino, pesquisa e produção técnico-científica. 
Entre os anos de 2009 e 2015, 8.360 pessoas foram atendidas no hospital lócus da pesquisa para diagnóstico e tratamento de câncer. A amostra final (n) foi composta por 150 prontuários de pessoas com câncer de mama que progrediram com feridas neoplásicas. A Tabela I descreve os dados sociodemográficos, em que se observou maior predominância de mulheres $(98,0 \%)$ na faixa etária entre 20 e 59 anos $(66,0 \%)$, de cor parda $(46,0 \%)$, casadas $(41,3 \%)$, com prole de um até três filhos $(40,6 \%)$, ensino fundamental incompleto $(24,0 \%)$, agricultoras $(25,3 \%)$ e aposentadas $(24,7 \%)$. Abaixo, seguem os dados da Tabela I.

Tabela I - Distribuição de prontuários de pessoas com feridas neoplásicas na mama segundo as características sociodemográficas dos pacientes. Campina Grande/PB, Brasil, 2016 ( $N=$ 150).

\begin{tabular}{|c|c|c|}
\hline Variável & $\mathbf{N}$ & $\%$ \\
\hline \multicolumn{3}{|l|}{ Sexo } \\
\hline Masculino & 3 & 2,0 \\
\hline Feminino & 147 & 98,0 \\
\hline \multicolumn{3}{|l|}{ Idade } \\
\hline $20-59$ & 99 & 66,0 \\
\hline$\geq 60$ & 51 & 34,0 \\
\hline \multicolumn{3}{|l|}{ Raça } \\
\hline Caucasiana & 49 & 32,7 \\
\hline Amarela & 14 & 9,3 \\
\hline Parda & 69 & 46,0 \\
\hline Negra & 1 & 0,7 \\
\hline Não especificado & 17 & 11,3 \\
\hline \multicolumn{3}{|l|}{ Estado civil } \\
\hline Solteiro & 27 & 18,0 \\
\hline Casado & 62 & 41,3 \\
\hline Viúvo & 13 & 8,7 \\
\hline Divorciado & 16 & 10,7 \\
\hline Não especificado & 32 & 21,3 \\
\hline \multicolumn{3}{|l|}{ Número de filhos } \\
\hline Nenhum & 13 & 8,7 \\
\hline 01 a 03 & 61 & 40,6 \\
\hline 04 a 05 & 13 & 8,7 \\
\hline Mais de 05 & 13 & 8,7 \\
\hline Não especificado & 50 & 33,3 \\
\hline \multicolumn{3}{|l|}{ Escolaridade } \\
\hline Sem escolaridade & 26 & 17,3 \\
\hline Ensino fundamental incompleto & 36 & 24,0 \\
\hline Ensino fundamental completo & 24 & 16,0 \\
\hline Ensino médio completo & 18 & 12,0 \\
\hline Ensino superior completo & 6 & 4,0 \\
\hline Não especificado & 40 & 26,7 \\
\hline \multicolumn{3}{|l|}{ Profissão } \\
\hline Agricultor & 38 & 25,3 \\
\hline Do lar & 33 & 22,0 \\
\hline Outros $^{1}$ & 26 & 17,3 \\
\hline Não especificado & 53 & 35,4 \\
\hline \multicolumn{3}{|l|}{ Ocupação } \\
\hline Empregado & 6 & 4,0 \\
\hline Aposentado & 37 & 24,7 \\
\hline Autônomo & 11 & 7,3 \\
\hline Não especificado & 96 & 64,0 \\
\hline TOTAL & 150 & 100,0 \\
\hline
\end{tabular}


A Tabela II, logo abaixo, dispõe sobre os dados clínicos da doença dos pacientes com feridas neoplásicas. Ao analisá-la, constatou-se que o tipo histológico mais incidente do câncer de mama foi o carcinoma ductal invasivo correspondendo a $87,3 \%$ da amostra. No que se refere ao tempo do diagnóstico, $83(55,4 \%)$ prontuários informavam que o diagnóstico de câncer de mama foi referenciado em menos de seis meses até o início do tratamento. Em relação à extensão da doença, o tumor local se sobressaiu, estando presente em 98 (65,3\%) pacientes. Quanto às doenças associadas, $112(74,7 \%)$ dos prontuários não possuíam essa informação, contudo, $22(14,7 \%)$ pessoas apresentaram apenas uma doença associada ao diagnóstico do câncer, em que a hipertensão arterial sistêmica (HAS) se sobressaiu.

No tocante aos sinais e sintomas, $82(54,7 \%)$ prontuários não apresentaram esta informação, entretanto 49 (32,7\%) pacientes apresentavam múltiplos sintomas em decorrência do câncer. Ressalta-se que dentre os sinais e sintomas mais prevalentes neste estudo, destacaram-se: náuseas, vômitos, diarreia, alopecia, astenia, disfagia, descamação, constipação, edema e hiperemia. No que concerne à reincidência do câncer, 107 (71,3\%) prontuários não especificavam esse dado e $31(20,7 \%)$ pessoas não apresentaram recidiva do tumor. Outrossim, quanto ao desfecho clínico, 141 (94,0\%) prontuários não possuíam esse dado, no entanto nove $(6,0 \%)$ prontuários apresentavam registro de alta hospitalar.

Tabela II - Distribuição de prontuários de pessoas com feridas neoplásicas na mama segundo as características clínicas da doença. Campina Grande/PB, Brasil, $2016(N=150)$.

\begin{tabular}{|c|c|c|}
\hline Variável & n & $\%$ \\
\hline \multicolumn{3}{|c|}{ Tipos histológicos do câncer } \\
\hline Carcinoma ductal invasivo & 131 & 87,3 \\
\hline Outros $^{2}$ & 19 & 12,7 \\
\hline \multicolumn{3}{|l|}{ Tempo de diagnóstico } \\
\hline Menos de 06 meses & 83 & 55,4 \\
\hline 06 a 12 meses & 41 & 27,3 \\
\hline Mais de 12 meses & 26 & 17,3 \\
\hline \multicolumn{3}{|l|}{ Extensão da doença } \\
\hline Tumor local & 98 & 65,3 \\
\hline Disseminação regional & 38 & 25,4 \\
\hline Disseminação distante & 14 & 9,3 \\
\hline \multicolumn{3}{|l|}{ Doencas associadas } \\
\hline $\mathrm{Uma}^{3}$ & 22 & 14.7 \\
\hline Múltiplas ${ }^{4}$ & 16 & 10,6 \\
\hline Não especificado & 112 & 74.7 \\
\hline \multicolumn{3}{|l|}{ Sinais e Sintomas } \\
\hline $\mathrm{Um}^{5}$ & 19 & 12,6 \\
\hline Múltiplos ${ }^{6}$ & 49 & 32,7 \\
\hline Não especificado & 82 & 54,7 \\
\hline \multicolumn{3}{|l|}{ Reincidência } \\
\hline $\operatorname{Sim}$ & 12 & 8,0 \\
\hline Não & 31 & 20.7 \\
\hline Não especificado & 107 & 71,3 \\
\hline \multicolumn{3}{|l|}{ Desfecho Clínico } \\
\hline Alta & 9 & 6,0 \\
\hline Óbito & - & - \\
\hline Não especificado & 141 & 94,0 \\
\hline TOTAL & 150 & 100,0 \\
\hline \multicolumn{3}{|c|}{$\begin{array}{l}{ }^{2} \text { Carcinoma Ductal Cribiforme; Carcinoma pouco diferenciado; Carcinoma Mamário Metastático; Carcinoma Papilar } \\
\text { Invasivo; Carcinoma Nuclear; Xantogranuloma. } \\
{ }^{3} \text { Hipertensão Arterial Sistêmica (HAS); Diabetes Mellitus (DM); Derrame Pleural; Herpes Labial. } \\
{ }^{4} \text { HAS + DM; Gastrite Crônica + Talassemia; HAS + DM + Mioma; Esquizofrenia + Déficit Visual e Auditivo; Erisipele + } \\
\text { Hérnia de Disco; Osteopatia + Esteatose Hepática + Hiperinsuflação pulmonar. } \\
{ }^{5} \text { Dor em diversas partes do corpo; Prurido. } \\
\text { 6áuseas + Vômitos + Diarreia; Alopecia + Febre; Cefaleia + Tosse + Parestesia; Astenia + Disfagia + Dispneia; } \\
\text { Descamação + Constipação; Edema + Hiperemia. } \\
\text { Fonte: Dados da pesquisa, 2016. }\end{array}$} \\
\hline
\end{tabular}


A Tabela III, que segue logo abaixo, destaca o perfil clínico das feridas neoplásicas com ênfase no aspecto da lesão e sinais e sintomas presentes. Ao analisá-la, percebe-se que em relação ao aspecto clínico da lesão, $53(35,4 \%)$ prontuários não continham essa informação, contudo a ulceração infiltrante estava presente em $46(30,7 \%)$ pacientes com feridas neoplásicas. Quanto aos sinais e sintomas, observa-se que a dor foi registrada em apenas 23 $(15,3 \%)$ prontuários, exsudato em $14(9,3 \%)$, sangramento em cinco $(3,3 \%)$, odor e sinais de infecção, igualmente, em quatro $(2,7 \%)$.

Tabela III - Distribuição de prontuários de pessoas com feridas neoplásicas na mama segundo as características clínicas da lesão. Campina Grande/PB, Brasil, $2016(N=150)$.

\begin{tabular}{lll}
\hline Variável & $\mathbf{n}$ & $\%$ \\
\hline Aspecto da lesão & 16 & 10,6 \\
Nódulo superficial & 6 & 4,0 \\
Nódulo profundo & 1 & 0,7 \\
Nódulo ulcerado & 16 & 10,6 \\
Ulceração superficial & 46 & 30,7 \\
Ulceração infiltrante & 8 & 5,3 \\
Ulceração vegetante & 1 & 0,7 \\
Multinodular & 3 & 2,0 \\
Outros & 53 & 35,4 \\
Não especificado & & \\
Sinais e Sintomas & & \\
Dor & 23 & 15,3 \\
Sim & 127 & 84,7 \\
Não especificado & & \\
Odor & 4 & 2,7 \\
Sim & 146 & 97,3 \\
Não especificado & & \\
Sangramento & 5 & 3,3 \\
Sim & 145 & 96,7 \\
Não especificado & & \\
Exsudato & 14 & 9,3 \\
Sim & 136 & 90,7 \\
Não especificado & & \\
Sinais de Infecção & 4 & 2,7 \\
Sim & 146 & 97,3 \\
Não especificado & $\mathbf{1 5 0}$ & $\mathbf{1 0 0 , 0}$ \\
\hline TOTAL & & \\
\hline IUlceracão infiltrante & & \\
\hline
\end{tabular}

'Ulceração infiltrante + Vegetante; Nodular Profunda + Ulceração infiltrante; Nodular superficial + Ulceração vegetante; Fonte: Dados da pesquisa, 2016.

Ademais, a Tabela IV destacada logo a seguir, exibe as modalidades terapêuticas utilizadas pelos pacientes com feridas neoplásicas, segundo as variáveis: procedimento cirúrgico, radioterapia, quimioterapia, tratamento medicamentoso para a dor e produtos utilizados na ferida. Nela, percebe-se que $63(50,7 \%)$ pacientes realizaram mastectomia total, $90(60,0 \%)$ pessoas se submeteram a $21-30$ sessões de radioterapia e $125(83,3 \%)$ realizaram quimioterapia. No que concerne ao tratamento medicamentoso para dor, havia registro de medicamentos utilizados apenas em 23 prontuários, contudo $17(73,9 \%)$ não especificava 0 medicamento, seguido de três $(13,0 \%)$ que utilizavam opioides fortes, dois $(8,7 \%)$, opioides fracos e um $(4,4 \%)$ analgésicos não-esteroidais. Quanto aos produtos utilizados na ferida, 145 $(96,7 \%)$ prontuários não continham essa informação, entretanto observa-se que dois $(1,3 \%)$ pacientes faziam uso de Sulfadiazina de prata, dois $(1,3 \%)$ óleo de girassol e um $(0,7 \%)$ trofodermin. 
Tabela IV - Distribuição de prontuários de pessoas com feridas neoplásicas na mama segundo as modalidades terapêuticas utilizadas durante a assistência. Campina Grande/PB, Brasil, $2016(N=150)$

\begin{tabular}{lll}
\hline Variável & N & $\%$ \\
\hline Procedimento cirúrgico & 58 & 38,7 \\
Biópsia & 23 & 15,3 \\
Retirada parcial do órgão & 63 & 42,0 \\
Retirada total do órgão & 6 & 4,0 \\
Reconstrução & & \\
Radioterapia & 37 & 24,7 \\
Não realizou & 3 & 2,0 \\
Até 10 sessões & 4 & 2,7 \\
$11-20$ sessões & 90 & 60,0 \\
$21-30$ sessões & 5 & 3,3 \\
Acima de 30 sessões & 11 & 7,3 \\
Não especificado & & \\
Quimioterapia & 125 & 83,3 \\
Sim & 25 & 16,7 \\
Não especificado & & \\
Tratamento medicamentoso para a dor & & \\
Analgésicos não opioides/AINES & 1 & 4,4 \\
Opioides fracos & 2 & 8,7 \\
Opioides fortes & 3 & 13,0 \\
Não especificado & 17 & 73,9 \\
Produtos utilizados na ferida & & \\
Sulfadiazina de Prata & 2 & 1,3 \\
Óleo de Girassol & 2 & 1,3 \\
Trofodermin & 1 & 0,7 \\
Não especificado & 145 & 96,7 \\
\hline TOTAL & $\mathbf{1 5 0}$ & $\mathbf{1 0 0 , 0}$ \\
\hline 8. & & \\
\hline
\end{tabular}

${ }^{8}$ Apenas 23 pacientes relataram sentir dor, conforme tabela III; Fonte: Dados da pesquisa, 2016.

\section{Discussão}

Analisando a tabela I, observa-se que a maioria $(98,0 \%)$ dos participantes da pesquisa é do sexo feminino. Essa circunstância pode ser explicada pelo fato do gênero feminino ser o principal fator de risco para o desenvolvimento de câncer de mama. No Brasil, excluindo-se os tumores de pele não melanoma, o câncer de mama é o tipo de neoplasia mais comum no sexo feminino em todas as regiões, exceto na região Norte, na qual o câncer do colo do útero ocupa o primeiro lugar [1].

Postula-se que a mulher tenha mais risco de desenvolver câncer de mama, devido à hipótese que os hormônios estrógeno e progesterona possam contribuir para o processo de desenvolvimento do câncer de mama, atuando como alimento para aquela célula que se encontra alterada. Atualmente, cerca de $60 \%$ dos casos de câncer de mama são estimulados pelos hormônios femininos, principalmente o estrogênio; assim entre os fatores de risco para câncer de mama estão: ser mulher e o processo de envelhecimento [8].

No que se concerne à idade, evidenciou-se que houve prevalência de feridas neoplásicas decorrentes do câncer de mama em mulheres com idade entre 20 e 59 anos $(66,0 \%)$. Estudo realizado com 12.689 mulheres com câncer de mama verificou que as mesmas apresentavam uma idade média entre 18 e 39 anos, o que demonstra que o câncer de mama está afetando cada vez mais mulheres jovens [9].

Em relação à raça, $69(46,0 \%)$ dos pacientes eram pardos. No Brasil, em 2014, a maioria $(53,6 \%)$ da população se dizia parda; no Nordeste, a maioria $(61,9 \%)$ das pessoas também se autodenominou parda. Estudos genéticos realizados em habitantes de capitais nordestinas têm confirmado a origem mestiça dessa população formada pela miscigenação de europeus, africanos e índios [10].

A predominância da raça parda do estudo $(46,0 \%)$ diverge de um estudo que encontrou maior proporção de casos de feridas em pessoas brancas $(80,6 \%)$, porém a porcentagem da raça caucasiana desta pesquisa $(32,7 \%)$ confirma a proporcionalidade da raça/cor para a 
população geral do Nordeste. Vale destacar que a variável raça/cor deste estudo não é autodeclarada [11].

No que concerne ao estado civil, $62(41,3 \%)$ das mulheres com feridas neoplásicas eram casadas. Esse dado é de grande relevância, uma vez que o companheiro tem papel fundamental no ajustamento ao diagnóstico e tratamento da doença. Outrossim, a presença do companheiro minimiza o risco de o paciente evoluir para quadros psíquicos, tais como ansiedade e depressão [6].

No quesito número de filhos, 61 pacientes $(40,6 \%)$ tinham entre um e três filhos. A contribuição dos filhos para a melhoria da qualidade de vida e bem-estar da pessoa com câncer está relacionada a vários aspectos, dentre eles destacam-se os cuidados com a higiene, alimentação específica, medicação, curativos, bem como manifestações de amor, afeto e solidariedade. Nesse sentido, o suporte da família no cuidado à pessoa com feridas neoplásicas contribui significativamente para o desenvolvimento do bem-estar físico, emocional e social daquele que padece da doença e da lesão [12].

De acordo com o Instituto Brasileiro de Geografia e Estatística (IBGE), o número de filhos por mulher diminuiu nos últimos 15 anos no Brasil, passando de 2,39 filhos/mulher no ano 2000 para 1,72, entre 2000 e 2015. Associado à queda na taxa de fecundidade, aumentou o percentual de mulheres sem filhos no país, fato que pode explicar os achados do estudo em questão. Diante disso, observa-se que, atualmente, as mulheres têm atrasado a maternidade e muitas optam por não ter filhos ou engravidam tardiamente, o que leva a compreender que estes fatores relacionados à vida reprodutiva da mulher também estão ligados ao risco de desenvolver esse tipo de neoplasia, uma vez que fatores como nuliparidade e primigestação tardia (após os 30 anos de idade) contribuem para aumento no risco do câncer de mama, haja vista que a amamentação em idade menor que 35 anos se associa a um menor risco de desenvolvimento de câncer de mama [1,13].

No que se refere ao item escolaridade, $40(26,7 \%)$ prontuários não possuíam essa informação, porém em $36(24,0 \%)$ havia a informação de que os participantes possuíam ensino fundamental incompleto. Pesquisa realizada com pacientes acometidos por feridas neoplásicas do Hospital do Câncer de Pernambuco revelou que a maior parte da amostra $(47,1 \%)$ possuía o ensino fundamental incompleto. Possivelmente, o menor grau de instrução influencie diretamente na progressão da doença e da lesão, uma vez que a pessoa pode possuir uma percepção reduzida do processo de saúde-doença e consequentemente, demorar a procurar atendimento médico para fins de diagnóstico e tratamento da doença, dificultando o acesso a terapêuticas mais avançadas, que poderiam elevar as chances de cura [14].

Em relação à profissão, pode-se constatar que, neste estudo, $53(35,4 \%)$ dos prontuários não especificavam a profissão dos participantes da pesquisa e 38 (25,3\%) pacientes eram agricultores. Ademais, no que concerne à ocupação, percebeu-se que a maioria $(64,0 \%)$ dos prontuários não continha essa informação, contudo $37(24,7 \%)$ dos pacientes eram aposentados.

Ao analisar a Tabela II, verifica-se que em relação ao tipo histológico mais incidente do câncer de mama, o carcinoma ductal invasivo sobressaiu em $131(87,3 \%)$ pacientes. Esse fato pode ocorrer devido à maioria (cerca de $80 \%$ ) dos tumores de mama se originar no epitélio ductal [1].

Estudo realizado com mulheres diagnosticadas com câncer de mama e tratadas nas unidades hospitalares de câncer do INCA e da Fundação Oncocentro de São Paulo (FOSP) apontaram que dos 12.689 casos analisados, 10.832 (90,7\%) foram diagnosticadas com carcinoma ductal invasivo, corroborando os achados do presente estudo [9].

No que se refere ao tempo do diagnóstico, $83(55,4 \%)$ prontuários informavam que o diagnóstico de câncer de mama foi obtido em menos de seis meses até o início do tratamento. Esse dado provavelmente se deve à procura rápida de ajuda médica pelos pacientes, uma vez que a Lei $\mathrm{n}^{\circ} 12.732 / 2012$ garante ao paciente com doença oncológica, o direito ao início do tratamento em um período igual ou inferior a 60 dias após confirmado o diagnóstico, bem como pelo fato do acesso a exames no Sistema Único de Saúde (SUS), assim como tratamento da doença ter sido ampliado gradativamente. No Nordeste, o principal aumento foi na faixa prioritária, ampliando em cinco vezes o número de mamografias realizadas, passando de 124 mil para 629.517. No geral, o número de exames saltou de 261.341 para 401.421 [15-16].

Em relação à extensão da doença, o tumor local se sobressaiu, estando presente em $98(65,3 \%)$ pacientes. Possivelmente, essa dimensão do tumor esteja relacionada com o início precoce do tratamento do câncer. 
Quanto às doenças associadas, $112(74,7 \%)$ prontuários não possuíam essa informação, contudo $22(14,7 \%)$ pacientes apresentaram uma doença associada ao diagnóstico do câncer, em que a HAS foi à comorbidade que se sobressaiu nesses pacientes. É imperioso destacar que a presença de comorbidades pode ser explicada pelo fato do câncer ser uma doença que compromete e debilita o paciente das mais variadas formas. Autores afirmam que a HAS é a comorbidade mais frequentemente registrada em pacientes com câncer e sua incidência aumenta com o tratamento quimioterápico, principalmente com a utilização de inibidores de angiogênese (IA). O mecanismo de ação dos IA em induzir HA não é completamente compreendido, mas pode estar diretamente relacionado à inibição da sinalização via tirosina quinase e dos fatores de crescimento endotelial vascular, em que essa inibição prejudica a vasodilatação [17].

No tocante aos sinais e sintomas, $82(54,7 \%)$ prontuários não apresentaram esta informação, entretanto 49 (32,7\%) pacientes apresentavam múltiplos sintomas em decorrência do câncer. Habitualmente, os sinais e sintomas do câncer estão relacionados à localização e ao tipo de tumor. A dor, náuseas e vômitos são relacionados à quimioterapia e são sintomas considerados desagradáveis pelos pacientes, bem como são determinantes na queda da qualidade de vida relacionada ao tratamento. Estudo realizado com 79 mulheres com câncer de mama verificou que $93 \%$ apresentaram náuseas e $87 \%$, vômitos, pelo menos uma vez durante o tratamento [18].

No que concerne à reincidência do câncer, 107 (71,3\%) prontuários não especificavam esse dado e 31 (20,7\%) pessoas não apresentaram recidiva do tumor. Além disso, quanto ao desfecho clínico, $141(94,0 \%)$ prontuários não possuíam esse dado, no entanto nove (6,0\%) prontuários apresentavam registro de alta hospitalar.

Neste contexto, um estudo realizado com 460 mulheres diagnosticadas com câncer de mama, atendidas em um hospital referência em oncologia, na cidade de Goiânia/GO, verificou que $213(46,3 \%)$ não apresentaram recidiva do tumor, $132(28,7 \%)$ mulheres possuíam recidivas e $115(25,0 \%)$ prontuários não apresentavam informações sobre esse dado, estando em consonância com o estudo em tela [19].

Ao verificar a Tabela III, constata-se que em relação ao aspecto clínico da lesão, 53 $(35,4 \%)$ prontuários não continham essa informação, contudo a ulceração infiltrante estava presente em $46(30,7 \%)$ participantes com feridas neoplásicas.

Do ponto de vista clínico, as lesões neoplásicas podem ser classificadas em nodulares, ulceradas e vegetantes. As nodulares caracterizam-se por serem recobertas de mucosa normal e podem ser benignas ou malignas; as ulceradas são divididas em superficial, quando se situam paralelamente ao epitélio e infiltrante, quando invadem os tecidos subjacentes; e as vegetantes se caracterizam pela exteriorização e são denominadas exofíticas [3,14].

Quanto aos sinais e sintomas, observa-se que a dor foi registrada em apenas 23 $(15,3 \%)$ prontuários, sem detalhamento da intensidade e características do episódio álgico. De acordo com a Organização Mundial da Saúde (OMS), a dor pode ser avaliada em vários aspectos. No que tange à intensidade, pode ser classificada em leve, moderada e intensa. Para atribuir valores a estes aspectos qualitativos, a OMS lançou mão de várias escalas, a fim de mensurar e atribuir um valor a dor verbalizada pelo paciente. Uma das escalas mais utilizadas é a numérica, em que a pontuação de 0 a 3 é considerada "dor leve", 4 a 7 , "dor moderada" e 8 a 10, "dor intensa" [20].

Estudo realizado com 62 pacientes com câncer de mama avançado em um Hospital de São Paulo revelou que, dentre os sinais e sintomas mais frequentes, a dor se sobressaiu de forma significativa em $20(32,2 \%)$ participantes, corroborando a pesquisa [11].

Em relação ao odor, dos 150 prontuários analisados, 146 (97,3\%) não possuíam esta informação, apenas quatro $(2,7 \%)$ apresentavam esse registro, contudo não havia a classificação deste sintoma. O odor é um sintoma constante no dia a dia do paciente com ferida neoplásica em estágio avançado da doença, tornando-se uma preocupação frequente e angustiante não só para o paciente, mas também para seus familiares e cuidadores, uma vez que pode provocar isolamento social, depressão, vergonha, constrangimento e falta de apetite, o que gera impacto negativo em sua qualidade de vida [5].

No que tange ao sangramento, observou-se que 145 (96,7\%) prontuários não apresentavam esse registro e somente cinco $(3,3 \%)$ continham essa informação, e não havia a classificação desse sinal. É importante enfatizar que o sangramento pode ocorrer durante a realização do curativo ou espontaneamente e que além das causas supracitadas pode ser ocasionado devido aos distúrbios de coagulação relacionados à doença ou ao tratamento. 
Além disso, a proliferação de células cancerígenas pode causar erosão de vasos sanguíneos adjacentes, ocasionando sangramento, que pode levar o paciente a óbito [11].

No que concerne ao exsudato, $136(90,7 \%)$ prontuários não possuíam este dado. Observou-se que apenas $14(9,3 \%)$ apresentaram esse registro, mas não havia informações acerca da quantidade. A presença de exsudato no leito da lesão é comum durante o processo inflamatório, porém níveis excessivos podem ter efeitos prejudiciais. Nas feridas neoplásicas, o exsudato resulta de processos inflamatórios e infecciosos decorrentes da liquefação do tecido necrótico e consequente ativação das proteases pelas bactérias que colonizam a lesão, bem como pelo fato do tumor ser hiperpermeável ao fibrinogênio e plasma [5,11].

Ademais, em relação à infecção, $146(97,3 \%)$ prontuários não especificavam este registro, sendo identificado apenas em quatro $(2,7 \%)$ prontuários, contudo não houve registros de informações específicas sobre esse sinal. Os episódios infecciosos, geralmente, estão relacionados à redução da resposta imunológica, a proliferação de microrganismos anaeróbios e tecido desvitalizado na ferida neoplásica, que podem ser verificados pela presença de sinais sistêmicos e locais como: febre, aumento do odor, da dor, da exsudação, da mudança de coloração do leito da ferida dentre outros $[5,11,14]$.

Por fim, ao examinar a Tabela IV, percebe-se que em relação ao procedimento cirúrgico, $63(42,0 \%)$ pacientes realizaram mastectomia total, com o objetivo de reduzir a incidência e melhorar a expectativa de vida de mulheres pertencentes a populações consideradas de alto risco. A cirurgia visa à retirada total da glândula mamária e é realizada de acordo com o quadro de cada paciente e em algumas pacientes pode ocorrer esvaziamento de nódulos linfáticos axilares. Geralmente, esse procedimento cirúrgico é quase sempre inevitável em fases avançadas da doença [21].

Estudo realizado com mulheres mastectomizadas verificou que este procedimento acarreta uma série de consequências de ordem física e emocional que impactam na qualidade de vida, provocando alterações nas suas relações familiares e sociais, bem como florescer sentimentos de impotência e de frustração [21].

No quesito radioterapia, dos 150 prontuários analisados, observou-se que 102 (68,0\%) pacientes realizaram esse tipo de tratamento, destes, $90(43,1 \%)$ se submeteram a $21-30$ sessões. A radioterapia constitui-se em uma modalidade terapêutica em que é aplicada uma dose pré-calculada de radiação por um determinado tempo a um volume de tecido que engloba o tumor, buscando erradicar todas as células tumorais com o menor dano possível às células normais circunvizinhas. Com este método de tratamento, pretende-se à erradicação do tumor, melhorar a autoestima e sobrevida do paciente [22].

No tocante à quimioterapia, em $125(83,3 \%)$ prontuários havia registros de uso de quimioterápicos. A quimioterapia consiste na utilização de medicamentos administrados continuamente ou com intervalos que variam de acordo com esquemas terapêuticos; atua de forma sistêmica e agem indiscriminadamente nas células (normais ou cancerosas), e pode ser utilizada isoladamente ou em combinação com outras modalidades de tratamento [23].

A radioterapia e quimioterapia são modalidades terapêuticas paliativas, que melhoram o controle dos sinais e sintomas decorrentes da ferida neoplásica, como dor, exsudato e sangramento, bem como reduzem o tumor [14].

Quanto ao tratamento medicamentoso para a dor, apenas $23(15,3 \%)$ dos 150 prontuários possuíam a informação que o paciente utilizava medicamentos para esse sintoma, todavia $17(73,9 \%)$ não especificavam o medicamento utilizado, seguido de três $(13,0)$ pacientes que faziam uso de opioides fortes, dois $(8,7 \%)$ de opioides fracos e um $(4,4 \%)$ de anti-inflamatórios não esteroidais (AINES).

A OMS criou um método específico de classificação da dor e de medicamentos que devem ser utilizados de acordo com sua intensidade, com a finalidade de orientar os profissionais de saúde na condução correta do alívio deste sintoma. A Escada Analgésica da OMS padroniza o tratamento analgésico da dor baseado em uma escada de quatro degraus conforme a intensidade de dor que o paciente apresenta e verbaliza. É imperioso destacar que ao analisar os dados da pesquisa, ficou explícito que a dor sentida pelos pacientes não é tratada conforme sua intensidade, uma vez que em nenhum prontuário havia o registro da intensidade da dor e os medicamentos correspondentes. Infere-se, dessa forma, que a dor não é avaliada corretamente e a condução terapêutica é subvalorizada pelos profissionais de saúde [20].

No que diz respeito aos produtos utilizados nas feridas, em 145 (96,7\%) prontuários, esta informação não estava especificada. Apenas cinco prontuários possuíam registros sobre os produtos utilizados nas lesões. Ao considerar esses dados, percebeu-se que dois $(1,3 \%)$ 
pacientes faziam uso de sulfadiazina de prata, dois $(1,3 \%)$ de óleo de girassol e um $(0,7 \%)$ de trofodermin.

A sulfadiazina de prata é recomendada nos casos de persistência do prurido, quando se observa áreas de hiperemia ao redor da ferida associada à pápulas esbranquiçadas, bem como nos casos de odor grau I [3].

O óleo de girassol é um produto originado de óleos vegetais poli-insaturados, composto fundamentalmente de ácidos graxos essenciais (AGEs) que não são produzidos pelo organismo, tais como: ácido linoleico, ácido caprílico, ácido cáprico, vitamina $\mathrm{A}$, E e lecitina de soja. Os AGEs são necessários para manter a integridade da pele e a barreira de água, cujo objetivo é promover quimiotaxia e angiogênese, manter o meio úmido e acelerar o processo de granulação tecidual, sendo indicado na prevenção e tratamento de dermatites, lesões por pressão, venosa e neurotrófica, bem como no tratamento de úlceras abertas com ou sem infecção [3].

Ressalta-se que não existem estudos que recomendam ou contraindicam a utilização deste produto nas feridas neoplásicas, porém é indispensável questionar-se sobre o uso de produtos cicatrizantes, que induzem a divisão celular para fins de reparação tecidual, uma vez que a recidiva do tumor pode ser consequência de uma única célula alterada.

Estudo realizado com mulheres acometidas por feridas malignas mamárias avançadas evidenciou que a sulfadiazina de prata e o AGE foram utilizados, porém em menor quantidade. Outro estudo mencionou a utilização de sulfadiazina de prata $(22,5 \%)$ seguida de AGE $(16,1 \%)$ nas lesões de mama de 62 mulheres com câncer de mama avançado [11,14].

É oportuno ressaltar que os cuidados das lesões neoplásicas seguem os mesmos preceitos do curativo ideal, todavia as feridas neoplásicas possuem características peculiares que devem ser prontamente avaliadas e tratadas, levando sempre em consideração curativo confortável, funcional e estético.

O estudo em tela possibilitou traçar o perfil sociodemográfico, clínico e terapêutico de pacientes com feridas neoplásicas decorrentes do câncer de mama atendidos em um hospital referência da Paraíba, sendo um estudo relevante, pois agrega conhecimento sobre a realidade de pessoas que convivem com feridas neoplásicas, seus aspectos epidemiológicos e o manejo clínico destas lesões.

Como limitação deste estudo, destacou-se a escassez de produções científicas nesta área, bem como a fragilidade dos registros de enfermagem, sobretudo no que se refere à avaliação e manejo clínico das feridas neoplásicas decorrente do câncer de mama, impedindo uma análise mais ampla da assistência prestada, visto que o registro contempla informações subjetivas e objetivas do paciente, constituindo-se como um dos mais importantes indicadores da qualidade da assistência prestada.

Nessa perspectiva, ao concluir este estudo, observou-se a necessidade de inserir, efetivamente, educação permanente nos serviços de saúde para que sejam implementados espaços de discussão com os profissionais sobre a importância e qualidade dos registros em prontuários, uma vez que estas informações facilitam a comunicação entre os membros da equipe multiprofissional; fornecem dados para a elaboração de planos de cuidados; auxilia no acompanhamento da evolução do paciente; constitui um documento legal, de caráter sigiloso e científico; facilita auditoria; bem como contribui para dados epidemiológicos, ensino e pesquisa na área de saúde; e ofertar educação em saúde aos pacientes, visando reduzir danos locais e sistêmicos.

Vale salientar ainda a necessidade de melhorias de políticas públicas no quesito acessibilidade aos exames de imagens das mamas, implementação de projetos educativos e informativos sobre o autoexame e de treinamento de profissionais voltados aos programas de rastreamento de câncer de mama, com vistas a incentivar a prevenção dessa doença.

Nesse ínterim, sugere-se que a Comissão de Pele da instituição construa e implemente um protocolo para avaliação e tratamento de feridas neoplásicas, em forma de check list, validado por expertises na área, a fim de facilitar e padronizar ações e registros de enfermagem.

Espera-se que este estudo possa contribuir para a comunidade científica e assistencial, enriquecendo o banco de dados, e desperte o interesse para o desenvolvimento de novos estudos neste âmbito, pois dessa forma será possível conhecer a clientela atendida com maior 
propriedade e identificar se os cuidados prestados, nos diferentes contextos, estão sendo satisfatórios diante destas pessoas que sofrem com lesões oncológicas.

1. Brasil. Ministério da Saúde, Instituto Nacional de Câncer José Alencar Gomes da Silva. Estimativas 2016: incidência do câncer no Brasil. Rio de Janeiro: Inca; 2016.

2. Martins CA, Guimarães RM, Silva RLPD, Ferreira APS, Gomes FL, Sampaio JRC et al. Evolução da mortalidade por câncer de mama em mulheres jovens: desafios para uma política de atenção oncológica. Rev Bras Cancerol 2013;59(3):341-9.

3. Brasil. Ministério da Saúde, Instituto Nacional do Câncer. Tratamento e controle de feridas tumorais e úlceras por pressão no câncer avançado: série Cuidados Paliativos. Rio de Janeiro: Ministério da Saúde; 2011.

4. Beh SY, Leow LC. Fungating breast cancer and other malignant wounds: epidemiology, assessment and management. Exp Rev Qual Life Cancer Care 2016;1(2):137-44. https://doi.org/10.1080/23809000.2016.1162660

5. Gethin G, Grocott P, Probst S, Clarke E. Current practice in the management of wound odour: an international survey. Int J Nurs Stud 2014;51(6):865-74.

https://doi.org/10.1016/j.ijnurstu.2013.10.013

6. Probst S, Arber A, Faithfull S. Malignant fungating wounds: the meaning of living in an unbounded body. Eur J Oncol Nurs 2013;17(1):38-45. https://doi.org/10.1016/i.ejon.2012.02.001

7. Azevedo IC, Costa RKS, Holanda CSM, Salvetti MG, Torres GV. Conhecimento de enfermeiros da Estratégia Saúde da Família sobre avaliação e tratamento de feridas oncológicas. Rev Bras Cancerol 2014;60(2):119-27.

8. Brasil. A.C. Camargo Câncer Center - Centro de diagnóstico, tratamento, ensino e pesquisa [Internet]. 2018. [citado 2017 Dez 12]. Disponível em: http://www.accamargo.org.br/newsletter-atuacao/entenda-a-relacao-dos-hormonioscom-o-desenvolvimento-do-cancer-de-mama/355/

9. Pinheiro AB, Lauter DS, Medeiros GC, Cardozo IR, Menezes LM, Souza RMB, et al. Câncer de mama em mulheres jovens: análise de 12.689 casos. Rev Bras Cancerol 2013;59(3):351-9.

10. Brasil. Instituto Brasileiro de Geografia e Estatística. Estimativas de população 2015. Rio de Janeiro: IBGE; 2015.

11. Gozzo TO, Tahan FP, Andrade M, Nascimento TG, Prado MAS. Ocorrência e manejo de feridas neoplásicas em mulheres com câncer de mama avançado. Esc Anna Nery 2014;18(2):270-6.

12. Reyonlds $H$, Gethin $\mathrm{G}$. The psychological effects of malignant fungating wounds. Ewna J 2015;15(2):29-32.

13. Brasil. Instituto Brasileiro de Geografia e Estatística. Projeção da população do Brasil: taxa de fecundidade total no período de 2000 a 2015. Rio de Janeiro: IBGE; 2016.

14. Lisboa IND, Valença MP. Caracterização de pacientes com feridas neoplásicas. Estima 2016;14(1):21-8.

15. Brasil. Ministério da Saúde. Lei n¹2.732, de 22 de novembro de 2012. Dispõe sobre o primeiro tratamento de pacientes com neoplasia maligna comprovada e estabelece prazo para seu início. Brasília: Ministério da Saúde; 2012.

16. Brasil. Ministério da Saúde. Instituto Nacional do Câncer. Brasil avança no diagnóstico do câncer de mama. Rio de Janeiro: INCA; 2016.

17. Souza VB, Silva EN, Ribeiro ML, Martins WA. Hipertensão arterial no paciente com câncer. Arq Bras Cardiol 2015;104(3):246-52.

18. Gozzo TO, Moyses AMB, Silva PR, Almeida AM. Náuseas, vômitos e qualidade de vida de mulheres com câncer de mama em tratamento quimioterápico. Rev Gaúcha Enferm 2013;34(3):110-6. https://doi.org/10.1590/S1983-14472013000300014

19. Peres VC, Veloso DLC, Xavier RM, Salge AKM, Guimarães JV. Câncer de mama em mulheres: recidiva e sobrevida em cinco anos. Texto Contexto Enferm 2015;24(3):7407.

20. Wiermann EG, Diz MPED, Caponero R, Lages PSM, Araújo CZS, Bettega RTC et al. Consenso brasileiro sobre manejo da dor relacionada ao câncer. Rev Bras Oncol Clín 2015;10(38):132-43. 
21. Jesus MV, Soratto MT, Ceretta LB, Schawalm MT, Zimermann KCG, Dagostim VS. As vivências da mulher com câncer frente a mastectomia. Rev Saúde Com 2013;9(3):195206.Simões AR, Eiras M, GrillolM. Desvio de posicionamento em radioterapia para patologias de cabeça e pescoço e próstata: revisão de literatura. Rev Saúde Tecnologia 2012;8:24-30.

22. Cruz FS; Rossato LG. Cuidados com o paciente oncológico em tratamento quimioterápico: o conhecimento dos enfermeiros da Estratégia Saúde da Família. Rev Bras Cancerol 2015;61(4):335-41. 